\title{
On the Past and Future of Natural Philosophy
}

\author{
by Walter Noll *
}

The Oxford English Dictionary gives the following definition of Natural Philosophy:

"The study of natural bodies as such and the phenomena connected with them; physical science, physics."

The Encyclopaedia Britannica (1966 edition) has the following description:

“.... the term 'natural philosophy', which is a rendering of Aristotle's 'physics', was appropriated in the 17th century to the new natural science of Galileo and Newton... Apparently this usage continued in England when it had become obsolete in other countries. And even now there are survivals of this usage......and there are professors of 'natural philosophy' who are engaged in nuclear research. ”

The most famous title containing the term is, of course, Newton's Philosophiae Naturalis Principia Mathematica, published in 1687. The term was used, much later, in the title Treatise on Natural Philosophy by Kelvin and Tait, published in 1876 and reprinted in 1879.

Until perhaps 150 years ago, European universities had at most 4 branches of learning (facultas in Latin), namely Medicine, Law, Theology, and Philosophy. In the 17th century it became customary to make a distinction between "Natural Philosophy" and "Moral Philosophy". The splitting of Natural Philosophy into Physics, Chemistry, Biology, etc. is of much more recent origin. In the USA, the term Ph.D (Philosophiae Doctor) is still used for doctorates not only in these fields, but even in engineering and economics. In Germany, however, more specific terms such as Dr.Rer.Nat. and Dr.Ing. are now in use.

Instruction in engineering at the university level did not have its origin in the traditional universities. The beginning of the 19th century saw the founding of institutes of technology such as the ...cole Polytechnique in Paris and the Technische Hochschule in Berlin. (John Roebling, the designer of the Brooklin Bridge, had his training in the latter.) Now there are many such institutes all over the world, for example MIT, Cal.-Tech., and indeed the Politectico di Bari, the sponsor of this meeting. The distinction between these institutes and the traditional universities is gradually disappearing. Most of the traditional universities now have engineering departments and 
most of the institutes of technology now offer instruction in the natural sciences, the social sciences, and the liberal arts. In Germany and Austria the term "Technische Hochschule" has been replaced by "Technische Universit\%ot". When I arrived in Pittsburgh in 1956, I was employed at the Carnegie Institute of Technology; it is now called Carnegie Mellon University.

Clifford Truesdell, having been a historian of science, probably could have given a much better and more complete history of the term and meaning of Natural Philosophy than I can.

I note that, since 1998, the Radboud Universiteit Nijmegen in Holland has a Center for Medieval and Renaissance Natural Philosophy. The Center describes its aims and scope as follows: "The RU Center for Medieval and Renaissance Natural Philosophy (CMRNP) is a research center which promotes the study of natural philosophy and science in its formative period between the late Middle Ages and the seventeenth century. It is embedded in the Department of Ancient and Medieval Philosophy of Nijmegen."

Clifford Truesdell revived the terms Natural Philosophy and Rational Mechanics more than 50 years ago. How this came about is best described by quoting from his Method and taste in natural philosophy, the last of his collection Six Lectures on Modern Natural Philosophy [SL], published in 1966:

"... the revival of both of these old terms began as a measure of defense ... In 1946 I was employed as an adjunct to a large captured wind tunnel, where my interest was directed to rarefied gases. The gaseous chief, more dense than rare, was unable to place my efforts in any pigeonhole. With the professional certainty of a former assistant professor of physics in a minor degree mill, he knew what I did was not physics. While his senior aerodynamicist assured him it was just the purest of pure mathematics, an aging estimator of 'eigenvalues' begged to be relieved from evaluating my work on the ground that he himself was a mathematician. Indeed, several mathematician friends told me that any paper in which the words 'stress' or 'vorticity' appeared was clearly engineering or physics."

"It is pleasant to afford the luxury of being an eccentric, but no one likes to be nothing. I sought a name that would reflect mathematical approach to problems of the motion of masses and found it, naturally enough, in the designation that Newton used for his own work in this area. The term 'rational mechanics', coined by the ancients, was discarded in English only when science fell into professions * . Far from seeking to exclude any area, use of the term 'rational mechanics' indicates an interest 
broader than any of today's specialties, but no less precise. As time went on, the methods and views developed in rational mechanics proved useful in thermodynamics, electromagnetism, and relativity, so a still broader term of equal age and standing was sought and found in 'natural philosophy', which includes all the mathematical sciences of natural phenomena."

Here are some more quotes from the same lecture, making more succinct Truesdell's definition of modern natural philosophy:

"The first aim of modern natural philosophy is to describe and study natural phenomena by the most fit $^{* *}$ mathematical concepts. The most fit need not be the most modern, but they may be; indeed, since we are all, whatever our labels, actors on a common stage, they are likely to be. In paraphrase of the program of Kelvin \& Tait in their Treatise on Natural Philosophy, we neither seek nor avoid the most abstract mathematics. To use modern mathematics when it is appropriate, one must first acquire it and then see where it applies. Neither to learn nor to use it is easy, ...."

"The second difference in method lies deeper. Most physical scientists regard mathematical treatment as belonging only to a later stage in the development of a theory. While they may appreciate the need for mathematics, perhaps even quite fancy 'pure' mathematics, in solving specific problems, they regard the basic principles of the theory as originating in intuition, experiment, or higher authority. .... For such a man, mathematics is a device for calculating examples, but it plays no part in discovering the physical theory. .... In modern natural philosophy, the physical concepts themselves are made mathematical at the outset, and mathematics is used to formulate theories."

Here is a relevant quote from Truesdell's first lecture" of the Six Lectures [SL] cited above, given in 1963 and entitled Rational Mechanics of Materials:

"In all of natural philosophy, the most deeply and repeatedly studied part, next to pure geometry, is mechanics. The resurgence of rational mechanics, after half a century of drowsing, has signalled and led the rediscovery of natural philosophy as a whole, just beginning in our time."

"The science we call to our aid in constructing instruments with which to see nature is mathematics. Rational mechanics was the first domain of natural philosophy on which modern mathematics was brought to bear so as to form a real theory, comparable in generality and precision to classical geometry."

"Just as some experimental apparatus grows obsolete and can be 
forgotten, much of the mathematical apparatus of our grandfathers' time, especially that which physicists and engineers usually insist is essential 'applied mathematics', is in fact poor dull stuff the theorist today can safely cast aside in favor of the sharper tools of modern algebra and analysis. Newton said, 'Nature is simple, and affects not the pomp of superfluous causes.' To deal with general response we must learn to think simply again, to use mathematical concepts that represent experience unblunted and unblurred *"

To summarize: In the 17th and 18th century, mathematics and physics were not the separate specialties that they are today, and Natural Philosophy was the term used for the endeavor to understand nature by using conceptual mathematical tools.

As mentioned before, perhaps the most important scientific work of that era is Newton's Philosophiae Naturalis Principia Mathematica. He invented differential calculus, with new mathematical concepts, a new terminology, and new notations, which made possible a concise formulation of the laws of particle mechanics now named after him. ${ }^{* *}$

Euler, the most important natural philosopher of the 18th century, further developed the language of differential and integral calculus and used it as a conceptual tool for creating, for example, his theories of rigid bodies and perfect fluids. In 1741 he wrote : "....the usefulness of mathematics, commonly allowed to its elementary parts, not only does not stop in higher mathematics, but is in fact so much greater, the further that science is developed."

Another example of great natural philosophy is Maxwell's theory of electromagnetism of 1873, which could not have been formulated without what was, at the time, very sophisticated mathematics.

Not every important scientific advance requires advanced mathematics, even in physics. For example, Einstein's special theory of relativity of 1905 used very little mathematics, although it was, of course, a conceptual leap of historic significance. A mathematical conceptual analysis of Einstein's ideas was supplied later, in 1908, by Minkowski's concept of space-time.

One of the greatest feats of natural philosophy in all of history was Einstein's general theory of relativity of 1915. It could not have been formulated without the theory of abstract manifolds, started by Riemann in 1854. In 1905, Einstein knew nothing about that, but he realized that he had to learn it. Fortunately, he had the help of Hilbert, who was perhaps the 
greatest mathematician in the world at that time.

Of course, there are many more people, famous and not so famous, who have done excellent work in the spirit of natural philosophy as defined by Clifford Truesdell. Here are some names that come to mind: the Bernoullis, Lagrange, Cauchy, Stokes, Gibbs, Kirchhoff, Boltzmann, PoincarÈ, Lorentz, Weyl, Von Neumann. You surely can add more.

In the 1960's, Clifford Truesdell tried, and to some extent succeeded, to revive not only the term Natural Philosophy but also the spirit behind it. In 1962, he became the editor of a series of books called Springer Tracts in Natural Philosophy. He was the driving force behind the founding, in 1963, of the Society for Natural Philosophy. (I was one of the founding members.) The Society has experienced a vibrant life for many years. In the mid-1990s Clifford Truesdell's health declined and he was no longer able to maintain his leadership. The series Springer Tracts in Natural Philosophy was discontinued. In 1994, the Springer Verlag reneged on a promise to publish, in this series, a 239 page manuscript entitled Mathematical Structures of Special Relativity [MN], written by V. Matsko and me. The Society for Natural Philosophy was almost dissolved. This development was forshadowed by Clifford Truesdell in the lecture Method and taste in natural philosophy in [SL] cited above:

"Natural philosophy, scarcely reborn, must defend itself against attacks from without and corruption from within. On the outside, the high priests of religion science * threaten holy war against any apostle of reason. Within, there are those who would make natural philosophy one more of the trade unions of science."

Needless to say, I am extremely pleased that the Society for Natural Philosophy is in the process of being revived, and I am honored that I have been selected, with this lecture, to contribute to this revival. I am also pleased that there is now at least one member of the Society (John Ball) who has the title Professor of Natural Philosophy.

Before discussing the future of Natural Philosophy, I have to warn you that the term has recently been misused in several nefarious ways, which I found in a Google search. Here are some examples:

1) There is now an organization called The Natural Philosophy Alliance with the following description:

"The great majority of us are intensely critical of special relativity, general relativity, big bang theory, and Copenhagen quantum physics. 
Revision and/or replacement of Maxwell-Lorentz electrodynamics is a common theme. Most of us accept some type of an electromagnetic aether."

This "Alliance" is to genuine physics or natural philosophy as "Creation Science" is to genuine biology.

2) A book available at Amazon.com has the title Natural Philosophy: Written to Evolve the Human Consciousness. A review there describes this book as follows:

"A collection of crappy poems. It sounds like it could be an interesting read, but in reality (the author) has the writing talent of a 4 year old. .... "

3) Another book available at Amazon.com has the title The Natural Philosophy of Love, translated from French. It is a treatise on the sexual organs and sexual behavior of animals.

4) On the internet, there is now a Natural Philosophy discussion group, with the following description:

"What exactly is 'Natural Philosophy'? Well, the term is analogous to 'Physics', which is an area of science dedicated to understanding the fundamentals of the Universe we live in. Natural Philosophy can be seen as the bridge which joins science and philosophy; it is concerned both with what can be proved scientifically and speculated upon philosophically. "

When talking about the future of natural philosophy here, I use the term in Truesdell's sense, described best by repeating the following three quotations:

"The first aim of modern natural philosophy is to describe and study natural phenomena by the most fit mathematical concepts."

"In modern natural philosophy, the physical concepts themselves are made mathematical at the outset, and mathematics is used to formulate theories."

".... we must learn to think simply again, to use mathematical concepts that represent experience unblunted and unblurred."

Mark Twain, or maybe Yogi Berra or Groucho Marx, has said that it is very difficult to make predictions, especially about the future. I am sure 
that natural philosophy, in Truesdell's sense, has a future, and I do not doubt that this future will produce natural philosophy that compares in importance with Maxwell's electromagnetism and Einstein's general relativity. The fact that I am here gives me hope that the Society for Natural Philosophy will be involved. I cannot predict this future, but I can tell you what I think that some of it should look like.

The main interest of most of the present members of the Society for Natural Philosophy is in classical physics, mostly continuum mechanics and thermodynamics. I believe that too few members are interested, for example, in relativity or quantum mechanics. There are a few members who work on problems of biology in the spirit of natural philosophy. An example is Bernard Coleman's application of mechanics to investigate the shape of DNA molecules.

To describe my personal situation, let me quote Clifford Truesdell again, from the 1963 lecture Rational mechanics of materials in [SL] cited above:

"I said at the outset that Mr. Noll's theory of simple materials is simple and easy. 'Simple and easy' does not and should not mean 'expressed solely in terms of mathematics created 200 years ago and now taught to science students as an article of religion'. The main mathematical concepts used here are: manifold, smooth mapping, Euclidean space, vector, tensor, functional, and group. In each case, little more than the definition, or the concept itself, has been needed; the grinding operations commonly called 'mathematics' by physical scientists are of no use here. While more recently formalized, the concepts employed in Noll's theory are closer to common, untrained experience than are such things as Fourier coefficients, Laplace transforms, complex variables, Bessel functions, polynomial bases, etc., which many scientists seem to find more familiar."

Now I would like to quote Clifford Truesdell quoting me, in a lecture at the meeting of Society for Natural Philosophy held in Pittsburgh in 1993 at the occasion of my retirement from teaching:

"If another warrior in Valhall should praise one of Walter's achievements, he (Walter) will cry

but I don’t do it that way any more!†"

Truesdell's statement of 1963 above remains valid now, provided it is amended as follows: (The changes are indicated in italics.) 
Noll's new theory of simple materials is simple and easy. "Simple and easy" does not and should not mean "expressed solely in terms of mathematics created 60 years ago and now taught to science students as an article of religion". The main mathematical concepts used here are: sets, mappings, linear spaces, linear mappings, lineons (short for linear transformations), spaces of linear mappings, mathematical structures, isomorphisms, tensor functors, uniformities, ordered sets, linear cones. In each case, little more than the definition, or the concept itself, has been needed; the grinding operations commonly called "mathematics" by physical scientists are of no use here. While more recently formalized, the concepts employed in Noll's theory are closer to common, untrained experience than are such things as $R^{n}$, matrix algebra, formulas with indices and summation conventions, Sobolev spaces, minimizing sequences, etc., which many mathematical scientists seem to find more familiar.

Now, there is nothing wrong with Laplace transforms, complex variables, Sobolev spaces, and minimizing sequences, but they are of no use in the formulation of the theory. However, in my book, $R^{n}$, matrix algebra, and formulas with indices and summation conventions should be stamped out altogether.

In my view, the mathematical infrastructure for the future of natural philosophy should be based, in part, on the content of my 1987 book FiniteDimensional Spaces: Algebra, Geometry, and Analysis, Vol. I [FDS] (I am working on Vol.II now) and on the papers [NS], [NV], [N6], [N7], [N8], and [N9] listed in the References at the end.

Unfortunately, few people are comfortable with this infrastructure, and I have difficulty getting anything published when I use it. For example, I submitted an earlier version of the paper On Material Frame-Indifference to the Reviews of Modern Physics in 1995. I thought that this paper should be of interest to an audience wider than just those interested only in the mathematics of continuum physics. A letter from the editor of the Reviews, written in 1988, informed me that a 1961 paper by Bernard Coleman and me had become a citation classic and that he would welcome receiving other papers from me. However, my paper was rejected even though the editor conceded that "the article is clearly written". Here are some quotes from the reviewer:

"I enjoyed reading this paper and very much would like to see it 
published. I am afraid, however, that the Reviews of Modern Physics is not the appropriate place. I believe that the overwhelming majority of the readers of the journal will consider the paper unreadable. Not because the material presented is intrinsically difficult, but rather because the author's individual form of the 'Bourbakian' style is far removed from anything that physicists are willing to digest. .... Professor Noll is highly respected in the mathematical community and has more than once proved himself to be ahead of his time. ...."

The paper is now a part of the booklet described below.

After the Springer-Verlag reneged on the promise to publish the manuscript Mathematical Structures of Special Relativity by Vince Matsko and me, I received a letter from the Cambridge University press stating that they would be interested in publishing something by me. So I sent them the manuscript just mentioned. It was rejected, probably because the reviewer, an "expert" in relativity, could not understand our mathematics. I still don't know what to do with this manuscript.

In 1992 I wrote a paper ([N7] in the list of references) proposing a mathematical infrastructure that would be of use not only in continuum mechanics, but especially for the geometry of differentiable manifolds. I submitted it to the Bulletin of the American Mathematical Society. It was rejected with the following insulting comment from the referee: "One might tell him that elementary results couched entirely in his own non-standard notation won't be read by anyone." After an exchange of letters with the editor I concluded that it was useless to fight the dogmas of some branches of the mathematical establishment.

Recently, I wrote a booklet of 73 pages with the title Five Contributions to Natural Philosophy [FC]. Here is the table of contents:

[N0] Introduction

[N1] On the Illusion of Physical Space .

[N2] On Material Frame-Indifference.

[N3] Updating The Non-Linear Field Theories of Mechanics.

[N4] The Theory of Simple Semi-Liquids, a Conceptual

Framework for Rheology.

[N5] Nematic Semi-Liquids.

The back-cover of the booklet contains the following description: 
"Walter Noll was co-author, with Clifford Truesdell, of The NonLinear Field Theories of Mechanics'[NLFT]. It was first published in 1965 and has become the standard reference work in the field. It was reprinted in 1992, translated into Chinese in 2000, and again reprinted in 2004.

The Five Contributions to Natural Philosophy provide a blueprint for updating The Non-Linear Field Theories of Mechanics and hence a guideline for future developments in the field. It should be available next to The Non-Linear Field Theories of Mechanics in every scientific library."

I offered this booklet to the Springer-Verlag for publication, but the offer was rejected. It is available electronically as a PDF-file and may soon also be available at amazon.com.

The parts [N1] and [N2] of [FC] deal with the principle of material frame-indifference, which has been used tacitly and implicitly for a long time. Very often, however, it has been confused with material symmetry. In [N1] and [N2], I give it an explicit formulation using the mathematical infrastructure mentioned above, a formulation that makes confusion with material symmetry impossible.

I just finished a paper entitled A Frame-Free Formulation of Elasticity ([N9]). The Introduction to this paper contains this statement:

"The term 'principle of material frame-indifference' was introduced in 1965 by C. Truesdell and me in [NLFT]. Earlier, I had used the term 'principle of objectivity', and some people use this term to this day. I meant 'objectivity' to express independence of the 'observer', but Truesdell disliked the term as being too easily misinterpreted. In fact, I now believe that the principle has nothing to do with 'observer', who is defined in the dictionary to be a person. What matters is the use of frames of reference as a means to clarify the concepts of location and motion, as explained in Sect.4 of [N2] of [FC].*

Here is a full statement of the principle of material frameindifference as it applies to any physical system:

The constitutive laws governing the internal interactions between the parts of the system should not depend on whatever external frame of reference is used to describe them.

"The principle applies only to external frames of reference, not to 
frames that are constructed from the system itself. It is important to note that the principle applies only to internal interactions, not to actions of the environment on the system and its parts, because usually the frame of reference employed is actively connected with the environment. For example, if one considers the motion of a fluid in a container, one usually uses the frame of reference determined by the container, which certainly affects the fluid. Inertia should always be considered as an action of the environment on the given system and its parts, and hence its description does depend on the frame of reference used. .... It should be possible to make the principle of material frame-indifference vacuously satisfied by using an intrinsic mathematical frame-work that does not use an external frame-space at all when describing the internal interactions of a physical system. .... Here I will do just that for the classical theory of elasticity."

The principle of material frame-indifference is not a law of physics such as the balance law for forces, the law of gravitation, the law of inertia, or any constitutive law. It is merely a prescription for avoiding nonsense. I close with a relevant quote from Truesdell's 1963 lecture Rational mechanics of materials cited before:

"The principle of material indifference may be rendered plausible in several ways. For one thing, it is satisfied by every classical theory of materials, and also by most of the less familiar proposals of constitutive equations. For another, without such a principle a number of generally accepted ideas in physics would become meaningless. Consider, for example, what are called centrifugal forces. Take a spring, and on one end hang a weight of one pound. The spring lengthens, say by one inch. Now lay the spring on a horizontal table, fastening one end to the center, leaving the weight attached to the other end. Spin the table and adjust the angular speed until the spring again stretches exactly one inch. On seeing this demonstration in the laboratory, the freshmen, happy to participate in the experimental foundation of science, take it as obvious that the force exerted on the spring is again one poundal; substituting the appropriate numbers into the formula for centrifugal force, they are encouraged to join Galileo, Mach, and Leonardo Da Vinci as empiricists by calculating the angular speed that agrees with the measured value, to within experimental error. What has been assumed, tacitly, is that the elastic law or constitutive equation of the spring is invariant under rotation.... For an observer lacking this belief, the experiment measures nothing."

Acknowledgement: I am grateful to Roger Fosdick and Millard Beatty for 
helpful suggestions and proofreading.

\section{References:}

[SL] Truesdell, C.: Six Lectures on Modern Natural Philosophy, 117 pages, Springer-Verlag, 1966.

[FDS] Noll, W.: Finite-Dimensional Spaces: Algebra, Geometry, and Analysis, Vol. I, 393 pages. Kluwer Academic Publishers, 1987.

[FC] Noll, W.: Five Contributions to Natural Philosophy, 73 pages, 2004 Available as a PDF file at http://www.math.cmu.edu/ wn0g/noll

[NFLT] Truesdell, C. \& Noll, W.: The Non-Linear Field Theories of Mechanics, Encyclopedia of Physics, Vol. III/3, 602 pages. SpringerVerlag, 1965. Second Edition, 1992. Translation into Chinese, 2000. Third Edition, 2004.

[MN] Matsko, V. \& Noll, W.: Mathematical Structures of Special Relativity 239 pages, written in 1994.

[NS] Noll, W. \& Sch\%offer, J. J. : “Orders, gauge, and distance in faceless linear cones; with examples relevant to continuum mechanics and relativity”, Archive for Rational Mechanics and Analysis 66, 345-377 (1977).

[NV] Noll, W. \& Virga, E. G.: "Fit regions and functions of bounded variation”, Archive for Rational Mechanics and Analysis 102, 1-21 (1988).

[N6] Noll, W,: “A new mathematical theory of simple materials”, Archive for Rational Mechanics and Analysis 48, 1-50 (1972).

[N7] Noll, W.: "Isocategories and Tensor Functors”, 19 pages, 1992. Available as a PDF file at http://www.math.cmu.edu/ wn0g/noll 
[N8] Noll, W.: "The Geometry of Contact, Separation, and Reformation of Continuous Bodies”, Archive for Rational Mechanics and Analysis 122, 197-212 (1993).

[N9] Noll, W.: " A Frame-Free Formulation of Elasticity", 2005. Available as a PDF file at http://www.math.cmu.edu/ wn0g/noll 